\title{
The Experimental Method Study of Cultural Relic on 3D Digital Design

\author{
Licheng Zong
}

Northwest University, School of Arts, china 710069

175244567@qq.com

\begin{abstract}
Keywords: Cultural Heritage; Relics; Digitalization; Three-dimensional Scanning
\end{abstract}
\begin{abstract}
The digital design of cultural heritage provides a new method for the protection, inheritance and utilization of cultural heritage. Based on the digital design theory and method of Cultural Heritage, study the key technology of three-dimensional digital design for cultural heritage, a three-dimensional digital reconstruction method of cultural relics is constructed by computer aid, and proposed the idea of three-dimensional mesh texture mapping for two-dimensional image calibration, by the three-dimensional digitization experiment of the Bronze Bovine from late "Shang" dynasty, construct the process framework of cultural digital design completely, and the digital design theory and methods of cultural relics are verified.
\end{abstract}

\section{Introduction}

Cultural heritage is spiritual pillar of national, which embodies the national culture, economy, technology, science and technology and other aspects of the integration of the strength, it is necessary to protect the heritage that sustainable development and motivation. At present, the digital research on cultural heritage is mainly focused on the research of digital theory, model and system $^{[1]}$. For example, Zhao Dong ${ }^{[2]}$ studied the digital theory and feasibility of historical and cultural resources, and established the digital protection and development mode of cultural heritage in Shaanxi area. Tan Bi Yong ${ }^{[3]}$ study the digital system of cultural heritage on three aspects of technical, cultural and institutional. The paper [4] studied the basic method of digital technology in the digital collection, storage, restoration, reproduction, display and dissemination of intangible cultural heritage; The paper [5] studied the digital heritage of cultural heritage protection mechanism, the use of web technology to build Manchu non-heritage digital platform. The United States, Britain and Japan started earlier in the digitalization of cultural heritage, and digitized protection, conversion and dissemination of cultural heritage based on the rapid development of computer information technology. P. E. Debevec ${ }^{[6]}$ proposed a small amount of static image method based on the heritage modeling and rendering methods, combined with photo technology and three-dimensional algorithm to build a large material cultural heritage scene virtual modeling; Marc Levoy ${ }^{[7]}$ research the digital method for the Michelangelo digital display; Fotis Liarokapis ${ }^{[9]}$ studied the information exchange platform based on the enhancement of realistic technology, superimposed virtual multimedia learning content in realistic learning environment, and enhanced the digital display, learning and inheritance of cultural heritage. The key technology of digital design of cultural relics is mainly data acquisition, data processing, digital display and digital utilization.

\section{Digital Design Technology of Cultural Relics}

\section{Reconstruction Based on Scan Data}

In the process of three-dimensional scanning of cultural relics, it is necessary to establish the viewpoint of three-dimensional scanning ${ }^{[11]}$, the objects which is scanned often need to build 4,8 or more viewpoints, large scenes tend to reach dozens of viewpoints. These point of view to obtain the scanning point cloud relative to the viewpoint itself is a local coordinate, you need to point the point of view of the cloud data in order to accurately obtain the object's complete coordinate data. Before combine the point of view, need compression and denoising the point cloud data. Use the idea of polygon subdivision, for a point of $M \times N$ view within the 3D point cloud area: 
Step1: First calculate whether the points in the $M \times N$ constructed quadrilateral mesh are on the same plane;

Step2: Judgment: The same plane executes step3;Different plane execution step4;

Step3: Keep the four vertices in the plane of $M \times N$, delete all the other three points,finish;

Step4: The plane $M \times N$ is subdivided into four quadrilaterals;

Step5: Run step1。

During the scanning process, the scan itself needs to set the appropriate level of simplification and optimization, In the process of polygon subdivision compression, how to judge the denoising of the point cloud data of the scanned object is suitable according to the principle of average distance. It is only necessary to determine the average distance between the sampling points to determine whether the need to delete the extra point cloud data, scan the object point cloud data compression. The main step of the average distance is:

Step1: Define the sampling cube of longer $d$ and the streamline percentage of data points $\theta$;

Step2: Defines the average dot pitch cube, shown in Fig.1;

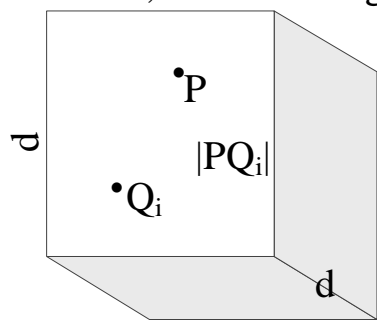

Figure 1. Sampling cube

Step3: Calculate the distance of any point from point $P$ to point $Q_{i}$

$|P Q|=\sqrt{\left(P_{x}-Q_{x}^{2}+\left(P_{y}-Q_{j}\right)_{y}+\left(P_{z}{ }^{2} C\right.\right.}$

Step4: Calculate the average distance, $\bar{D}=\frac{\sum_{i=1}^{n}\left|P Q_{i}\right|}{n}$

Step5: Contrast Reduction Percentage $\theta$, delete data points smaller than $\theta$.

After the above data acquisition and denoising processing, the data of cultural relics scanning point cloud are optimized. The three - dimensional model is reconstructed by the optimized 3D point cloud data to digitize the cultural relics.

\section{Digital Texture Mapping}

In the digital restoration of cultural relics, three-dimensional scanning records the spatial coordinates of cultural relics ${ }^{[12]}$, in order to accurately restore the complete information of cultural relics, in the three-dimensional scanning process, and simultaneous recording of high-precision texture information artifacts. According to the principle of perspective projection imaging, the three-dimensional scanning perspective projection model is used to match the grid model of cultural relics and image mark points, So that the coordinates of the cultural relic model are transformed into the camera coordinate system of the captured image, and the two-dimensional color image of the cultural relics is generated to make the texture mapping between the image and the model, the detailed steps are as follows:

Step1: Mark the point of $P$ of the artifacts on $(u, v)$;

Step2: Set the coordinates of the point $P$ on the cultural grid model be $(x, y, z)$;

Step3: So, $(u, v)$ and $(x, y, z)$ Called a set of feature correspondence, the relationship between them is expressed as follows: 


$$
\left|\begin{array}{l}
u \\
v \\
1
\end{array}\right|=M\left|\begin{array}{c}
x \\
y \\
z \\
1
\end{array}\right| \quad \text { among, } M\left|\begin{array}{cccc}
m_{11} & m_{12} & m_{13} & m_{14} \\
m_{21} & m_{22} & m_{23} & m_{24} \\
m_{31} & m_{32} & m_{33} & m_{34}
\end{array}\right| \text { is the perspective projection matrix; }
$$

Step4: mark $P_{n}$, acquire $M_{n}$ matrix, solve the perspective projection matrix;

Step5: Assign the calculated $P$ point RGB value to the mesh model;

Step6: Mark the main texture points of the artifacts, calculate Step2-Step5.

Digital reproduction of cultural relics not only requires accurate modeling of the three-dimensional model of cultural relics, but also requires realistic restoration of texture and color of cultural relics, depending on texture mapping. In the process of texture mapping, the color texture effect of the digital model of the cultural relic is related to the value of the mark point. The more the mark point is, the better the effect, but the calculation will increase sharply. Therefore, in the actual digitalization experiment of cultural relics, the combination of numerical calculation and artificial map is used to digitize the texture and color of the cultural relic, and it is convenient for the later digitization of cultural relics.

\section{Digitalization Experiment of Cultural Relics}

In order to test the effectiveness of the method above, the digital design experiment was carried out for bronze cattle. Experiments were performed using InSpeck 3D Scanner, the scanner through the parallel access to obtain data, access speed can reach 300,000 per second. In the digital experiment of bronze cow, the bronze cattle statue is scanned, the facets of the cultural relics are scanned, the three-dimensional point cloud data of bronze cattle are obtained, and the data processing and reconstruction are obtained to obtain the three-dimensional model and digital design application, experimental steps are as follows:

Step1:Experimental environment setting

In order to protect the cultural relics Bronze cow (Fig. 2) from harm, the light control of the laboratory at $300 \operatorname{lux}$ ( sunshine ) $+50 \mathrm{lux}$ (light), control the experimental environment humidity less than $40 \%$, the temperature is kept at about $23{ }^{\circ} \mathrm{C}$, and put the cultural relics on the scanning turntable, set the camera viewing angle and scan the turntable to maintain the horizontal angle, used to record the same angle of cultural relics texture photos.

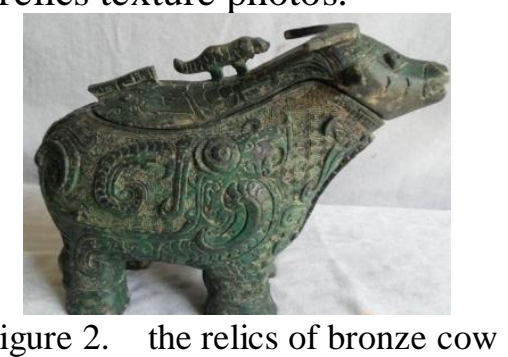

Step2:3D scan settings

Figure 2. the relics of bronze cow

In order to ensure the accuracy of the scan data, scan the scanner before the first calibration, set the scanning rate of $2.00 \mathrm{~mm}$, the number of positioning coordinates is 10 , the optimized scanning grid is 20 , and the simplified scanning grid is 10 , scanning texture projection mode is automatic, texture size is $4096 * 4096$.

Step3 Artifact scanning settings

As the main data design of the bronze cow respects the artifacts are located above the foot, the use of standing posture can scan $90 \%$ of the cultural data surface. Artifacts on the bottom of the data surface using artificial repair, so that artifacts can be avoided in the artifacts caused by injury, but also improve the progress of the experiment.

Step4: Acquisition of 3D scan data

The data of the bronze cow statue is scanned, and the three-dimensional coordinate information of the cultural relics (point cloud data) is obtained. During the scanning process, set the scanner's 
turntable rotation scale to $15^{\circ}$, the artifacts from the set of the original location of the beginning of scanning, each rotation $15^{\circ}$ a scan, a total of 24 scans, at the same time on the 24 times photo. Record 24 times the texture of the textured text shown in Fig. 3(B).

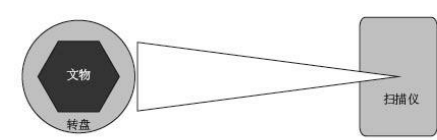

A

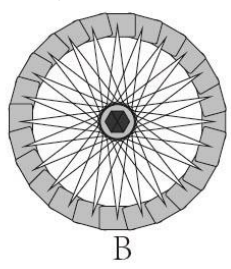

Figure 3. Scanning diagram

Step5: The processing of artifact scanning data

After 24 scans to obtain a large number of cultural relics 3D point cloud data, these point cloud data need to be processed to use. Using the average distance reduction method constructed above, the data of the obtained point cloud is simplified, the percentages of the definition of the purpose and purpose of the use of the cultural relics are shown in table 1.

Table 1 The percentage of data reduction

\begin{tabular}{|c|c|c|c|}
\hline purpose & $\operatorname{Simplify}(\%)$ & original & simplify \\
\hline Digital reduction & $53 \%$ & 117648 & 55295 \\
\hline Precision reduction & $26 \%$ & 117648 & 87060 \\
\hline Cultural relics show & $48 \%$ & 117648 & 61177 \\
\hline Film and animation & $64 \%$ & 117648 & 42354 \\
\hline
\end{tabular}

The purpose of the experiment is to digitally restore and re-design the bronze cemetery, so there is no need for a particularly high reduction accuracy. Therefore, the percentage of the definition is defined as 53\%, through experiments we can see that the bronze cow respect the original piece of 117648 , after the number of thin slices is 55295 .

Step6:Three - dimensional visual reconstruction of cultural relics

Through the three-dimensional reconstruction, a complete grid model of cultural relics is obtained, which is enough for ordinary three-dimensional display of cultural relics and digital design of cultural relics. So you can get step 5 effectiveness of the $53 \%$ reduction rate. At the same time, the obtained grid model is exported to FBX or OBJ format, which can be applied to most of the three-dimensional model software and virtual development platform, as shown in Fig. 4.

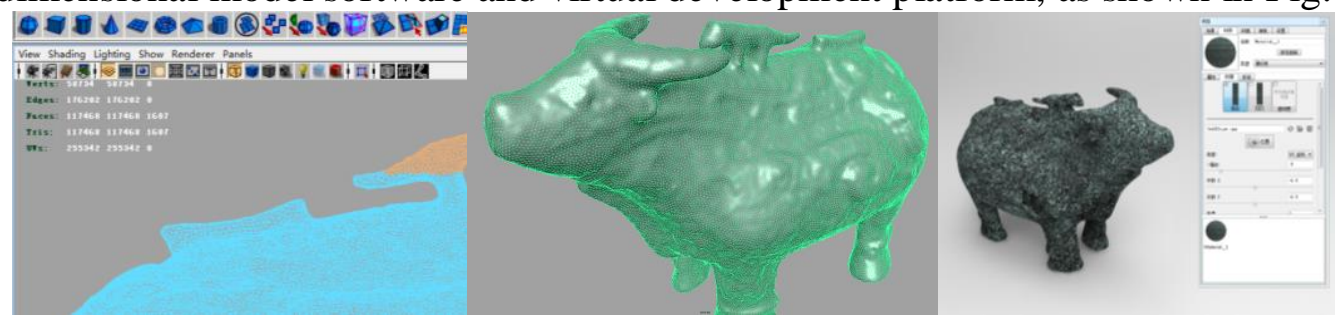

Figure 4. Three - dimensional model of cultural relics and statistics

Step7:Model texture mapping

In the course of the experiment, it was found that the experimental data was affected by the experimental environment. During the scanning process, the $15^{\circ}$ scan data was not affected, but the recorded texture information was affected by light. This part of the work needed to be modified later. Therefore, based on the above-mentioned perspective projection method, we selected $0{ }^{\circ}, 90^{\circ}$, $180^{\circ}, 270^{\circ}$ four images as the base pattern of texture mapping, and performed grid point calibration for two-dimensional images and three-dimensional model grid points of the registration, so as to obtain the texture of the three-dimensional model, the final results obtained as shown in Fig. 4. 


\section{Summary}

The three-dimensional digital design of culture involves the key technology of data acquisition and processing of cultural relics. The three-dimensional scanning method can obtain the three-dimensional coordinate information of cultural relics quickly and accurately, and reconstruct the three-dimensional model of cultural relics by processing the scanning data. Combined with the bronze cattle statue digital design experiment, to build a complete digital design process of cultural relics and experimental methods, compared with the three - dimensional digitalization of artifacts based on images and measurements, the three - dimensional digital experiment based on three dimensional scanning and computer - aided cultural relics can be used to obtain the three dimensional information of cultural relics more accurately and quickly.

\section{References}

[1] WEI liwei, LI wenwu. Research on Current Situation and Trend of Digital Culture Heritage Preservation Standards in China and Abroad[J]. China Standardization,2016(6): 91-96.

[2] ZHAO Dong. Research on the digital protection and development of historical cultural resources-with Shaanxi as the center[D]. Shangdong: Shan Dong University,2014.

[3] TAN Bi-yong. A comparative study of sino-foreign digital protection of intangible cultural heritage[J]. Library and Information, 2011(4):7-11.

[4] HUANG Yong-ling. Research on digital protection and development of intangible cultural heritage in China[J].Journal of Huazhong Normal University (Social Science),2012,51(2):49-55.

[5] DAI Jun-bo, SHAN Gui-hua. Design and implementation of digital protection platform for manchu intangible cultural heritage based on web[J]. Researches In Library Science,2010 (9) :32-34.

[6] Debevec P E, Taylor C J, Malik J. Modeling and rendering architecture from photographs: A hybrid geometry and image based approach[C]//Proceedings of the 23rd annual conference on Computer graphics and interactive techniques. ACM,1996:11-20.

[7] Levoy M, Pulli K,Curless B, et al. The digital michelangelo project: 3D scanning of large statues[C]/Proceedings of the 27th annual conference on Computer graphics and interactive techniques. ACM Press/Addison-Wesley Publishing Co., 2000:131-144.

[8] Lamers K, Eisenbeiss H, et al. Combining photogrammetry and laser scanning for the recording and modeling of the Late Intermediate period site of Pinchango Alto, Palpa, Peru[J].Journal of Archaeological Science,2010,34 (10) : 1702-1712.

[9] Liarokapis F, Petridis P, et al. Multimedia augmented reality interface for E-learning (MARIE)[J].World Transactions on Engineering and Technology Education,2002,1(2):173-176.

[10] HAN Dong-mei.Research on digital-protection technology of non-material cultural heritage based on Chinese-paper-cut[D] zhejiang:Zhejiang University, 2008.

[11]WANG Ru.Research on Key technologies of digital and three - dimensional modeling of ancient buildings[D]. shannxi:Northwest University,2010.

[12] ZHU Xiao-dong, ZHOU Ming-quan, GENG Guo-hua. Design and implementation of digital archaeology museum of Northwest University[J]. Journal of Northwest University (Natural Science Edition),2004,34(5):522-526. 